Faqi Liu, Zhidong Guan*, Tianya Bian, Wei Sun and Riming Tan

\title{
A novel analytical curved beam model for predicting elastic properties of 3D eight-harness satin weave composites
}

DOI 10.1515/secm-2016-0354

Received November 26, 2016; accepted January 28, 2017; previously published online April 21, 2017

Abstract: An offset representative unit cell (ORUC) is introduced to predict elastic properties of three-dimensional (3D) eight-harness satin weave composites both analytically and numerically. A curved beam model is presented based on minimum complementary energy principle, which establishes an analytical solution for elastic modulus and Poisson's ratio calculation. Finite element method is developed to predict engineering constants of composites. Modified periodic boundary conditions and load method for ORUC are also presented. Experiments of simulated material are performed under tensile test. Close correlation is obtained between experimental data and predictions. Sensitivity study is conducted and manifests that within a large variation of constitutive material properties, the curved beam model derives close predictions comparing to finite element model, which indicates the stability of the curved beam model. Parametric study is also conducted to discuss the effect of weave type and geometric dimensions on elastic properties. It is argued that the curved beam model could manifest fine predictions accurately and stably, and is recommended for the prediction of elastic properties of satin weave composite.

Keywords: complementary energy principle; eightharness satin weave; elastic properties; finite element method.

\section{Introduction}

Carbon/carbon composites (C/Cs) are regarded as one of the best thermal-structural materials due to their balanced properties in low density, high specific strength, good thermal stability and conductivity, low expansion

\footnotetext{
*Corresponding author: Zhidong Guan, Aircraft Department, Beihang University, Beijing 100191, People's Republic of China, e-mail: d5062010@163.com
}

Faqi Liu, Tianya Bian, Wei Sun and Riming Tan: Aircraft Department, Beihang University, Beijing 100191, People's Republic of China coefficient, and excellent ablation performances [1-3]. They are wildly used in aviation and aerospace fields where service temperature is super-high (over $3000^{\circ} \mathrm{C}$ ), such as reentry nose tips, rocket nozzles, and supersonic aircraft brakes [4, 5]. Unlike traditional laminated composites, C/Cs are usually structural material, especially three-dimensional (3D) weave $\mathrm{C} / \mathrm{Cs}$, which provide multidirectional reinforcement and directly enhance the stiffness and strength in the through-the-thickness direction. They do not possess weak planes of potential delamination and, hence, manifest increased impact resistance and fracture toughness $[6,7]$.

To take full advantage of these properties, it is necessary to investigate the mechanical behavior of the material and the component response into a deeper level. Considerable efforts have been made to understand their mechanical behavior, and many models have been developed to predict their stiffness and strength. These models are generally divided into analytical approaches [6-10] considering different assumptions of fabric geometry to calculate the mechanical properties of the composite and finite element methods [11-15] treating matrix and fibers as discrete components to obtain numerical results. Even though finite element methods seem more accurate, defining the fabric geometry and properties at each point inside a woven fabric composite is a complex and timeconsuming task, let aside the computational complexity. Now only a few detailed volumetric unit cell models have been created for the basic plain woven fabric composites; a generalized volumetric approach is not yet feasible. On the other hand, the finite element methods cannot be any more accurate unless truly representative boundary conditions are used [10]. In order to develop computationally efficient analysis tools for 3D composites, analytical approaches other than finite element methods are in clear demand in recent years.

The majority of analytical models are still rooting in classical laminate theory and orientation-averaging techniques. These models are based on the assumptions that the unit cell can be represented as an arrangement of unidirectional segments (with yarn orientations defined in 2D, not 3D space) and that iso-strain or iso-stress conditions apply. All of these are easy to implement and 
computationally inexpensive. Iso-stress models provide a lower bound to stiffness predictions, while iso-strain ones provide an upper bound [16]. The most representative works are made by Ishikawa and Chou [17] and Chou [18]. In the 1980s, they proposed three analytical models to predict elastic stiffness of woven fabrics based on classical thin laminate theory (CLT). In the mosaic model [18], a fabric composite is as an assemblage of pieces of asymmetrical cross-ply laminates; an iso-strain or iso-stress state is assumed to obtain the elastic stiffness matrices. In the fiber-undulation model [17], as an extension of the series model, fiber continuity and undulation (only in weft direction) are taken into consideration, and is adopted for modeling the "knee behavior" of plain woven fabric composites. This model is suitable for fabrics with lower-order repeats in the unit cell. The bridging model [17] is a combination of series and parallel models used in satin weave composites. It extends the fiber undulation model to model general satin weave composites, wherein there are straight thread regions surrounding an interlaced region, which are, thus, separated from one another.

A lot of researchers refined these models by considering more detailed constitutive geometry and properties. Aitharaju and Averill [11] considered fiber waviness in both directions and presented an analytical/numerical model. In the model, a unit cell was divided into three regions depending on tow waviness. By assembling these regions, the problem of estimating the Young's modulus and Poisson's ratio in all directions is divided into three subproblems, and the superposition method is used. Hallal et al. [19] takes into account the real geometry of undulated yarns and proposed an improved analytical model based on mixed iso-strain and iso-stress assembling models of a 2.5D interlock woven composite. According to this paper, it is proved that a model based only on an iso-strain condition could not give accurate results, while a mixed iso-strain and iso-stress model yields more accurate estimations.

Although refined CLT methods give improved predictions for several elastic properties, their applications are still limited within certain types of weaves and could only predict in-plane properties. In 1998, Gommers et al. [20] applied the Mori-Tanaka (M-T) method to different types of textile composites and concluded that especially with knitted fabrics, the M-T model yields better results than the classical iso-strain model.

Recently, a large amount of researchers have proposed models based on variational principles of strain energy. The strain energy method deals away with the iso-stress/ iso-strain assumption present in most analytical models, allowing for a more accurate prediction of the elastic properties, with a computational cost only a slight higher in comparison with CLT models. Tong et al. [21] assumed fiber yarn to be a curved beam supported by an elastic tension or shear foundation in a woven composite material. The curved beam modeling procedure is then utilized to predict the ultimate failure stress of the 3D orthogonal woven composite materials when subject to a tensile load in the filler direction. Sheng and Hoa [22] developed a 3D micro-mechanical model to obtain 3D stiffness and compliance matrices via variational potential energy method and variational complementary energy method. Xiong et al. [23] and Cheng and Xiong [24] proposed micro-mechanical models to predict the elastic modulus of 2D plain weave fabric (PWF) composite. The two orthogonal yarns in a micro-mechanical unit cell are idealized as curved beams with a path depicted using sinusoidal shape functions. The modulus is derived by means of a strain energy approach founded on micro-mechanics. Although the modulus of curved beams was derived directly, the existence of the matrix was neglected. As a substitute, a parallel model was employed afterward to obtain overall stiffness.

From the previous works, a lot of analytical models are developed for textiles, but only a few have been applied to satin weave composites. There is a need for analytical models to effectively predict the mechanical behavior of composites in a different weave geometry. Meanwhile, numerical techniques, such as the FEM, are also required to verify the validity of models.

In this paper, an offset representative unit cell (ORUC) is introduced to predict the elastic properties of 3D eightharness satin weave $\mathrm{C} / \mathrm{Cs}$ both analytically and numerically. A two-stage curved beam model is presented based on minimum complementary energy principle, which established an analytical solution for elastic property calculation. Finite element method is developed to predict engineering constants of $\mathrm{C} / \mathrm{Cs}$. Modified periodic boundary condition and load method for ORUC are also presented. Both analytical and numerical predictions are compared to experiment results as well as several existing models. A sensitivity study is conducted to verify the validity and stability of the analytical method. A parametric study is also conducted for a different weave type and geometric dimensions to discuss the effect of each parameter on elastic properties.

\section{Two-stage analytical curved beam model}

This section addresses the two-stage analytical curved beam model, which derives the elastic properties of 3D weave $\mathrm{C} / \mathrm{Cs}$. 
$\mathbf{A}$

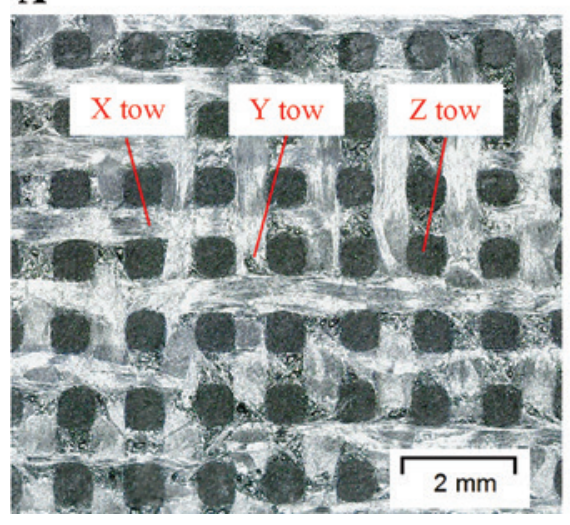

B

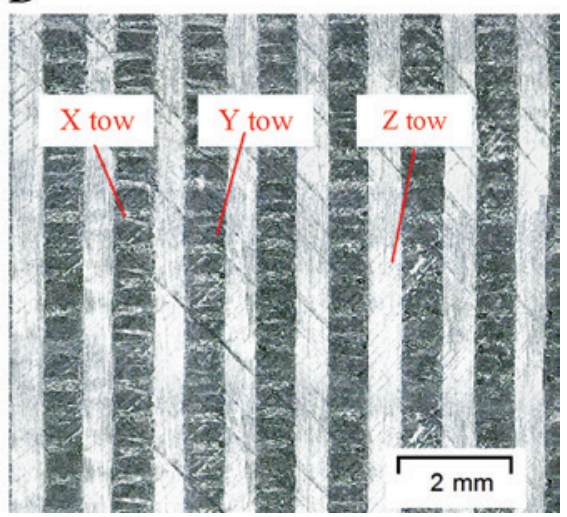

Figure 1: Micro-graphs of $C / C$ 3D eight-harness satin weave. (A) X-Y plane. (B) X-Z plane.

\subsection{Geometrical description and representative unit cell}

Figure 1 shows micro-graphs of $\mathrm{C} / \mathrm{C}$ 3D eight-harness satin weave composites. Figure $1 \mathrm{~A}$ is the section graph in the $\mathrm{X}-\mathrm{Y}$ plane, while Figure $1 \mathrm{~B}$ is the section graph in the $\mathrm{X}-\mathrm{Z}$ plane. It can be observed that the fabric in the X-Y plane is satin weave cloth where tows are undulated, while fiber tows in the $\mathrm{Z}$ direction are piercing tow, which are almost straight in composites.

From the micro-graphs, the geometry can be idealized as carbon fiber tows woven together as shown in Figure 2. The weave is geometrically balanced, which means that the geometry of warp and fill directions is identical [25].

Because of the geometric complexity of the eightharness satin weave composite, the representative unit cell (RUC) is usually selected as a full size cell [18], which includes 64 overlapping regions as shown in Figure 2. Yet, this RUC is quite complex, unlikely to perform neither analytical analysis nor numerical computation. Several researchers $[26,27]$ divided the RUC into three sub cells, which were used to assemble a full RUC by the rule of mixtures. However, as periodic boundary conditions are not suitable for sub cells, an iso-strain condition is usually applied, resulting in deviation from the reality. Herein, an offset representative unit cell (ORUC) is introduced in this paper to simulate the eight-harness satin weave composites, which should reduce the calculation complexity with functions equal to a full size cell. As shown in Figure 2, the part in the square box is selected to be the ORUC, which is only one eighth the volume of an ordinary RUC. ORUC repeats in non-vertical directions $\left(\overrightarrow{n_{1}}, \overrightarrow{n_{2}}\right)$ as shown in Figure 3 to form the whole material. As the ORUC in this paper differs from the traditional RUC reported in

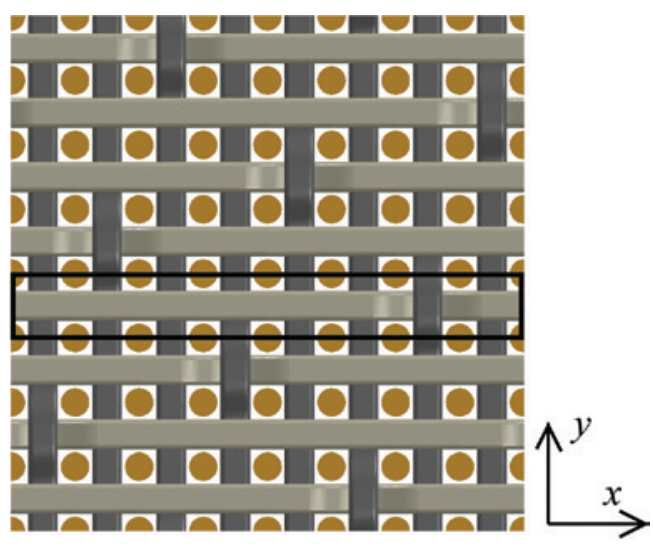

Figure 2: Woven fiber tows of C/C 3D eight-harness satin weave composites, traditional RUC (whole image), and ORUC (in square box).

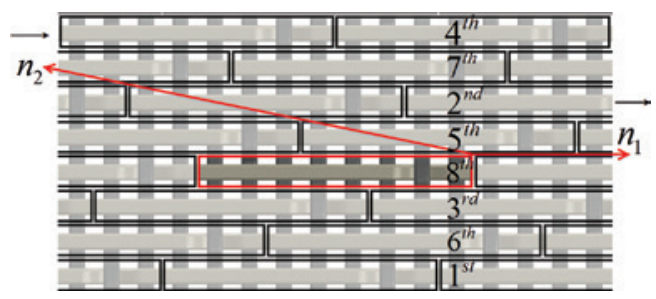

Figure 3: Repeating direction of periodic cell of weave composites (with matrix and $Z$ tows hidden).

other literatures $[18,17]$, a verification test is conducted in Appendix A. The structure of the ORUC is demonstrated in Figure 4. It should be noted that a matrix is hidden in Figure 4, and in the rest of this paper, fiber tows in different directions will be represented by $\mathrm{X}, \mathrm{Y}$, or $\mathrm{Z}$ tows referring to the coordinates demonstrated in Figure 4.

Because of repeatability of the ORUC, eight $Y$ tows are linked together in the sequence of 1st-6th-3rd-8th-5th-2nd4th (demonstrated in Figures 3 and 4), which exactly form 


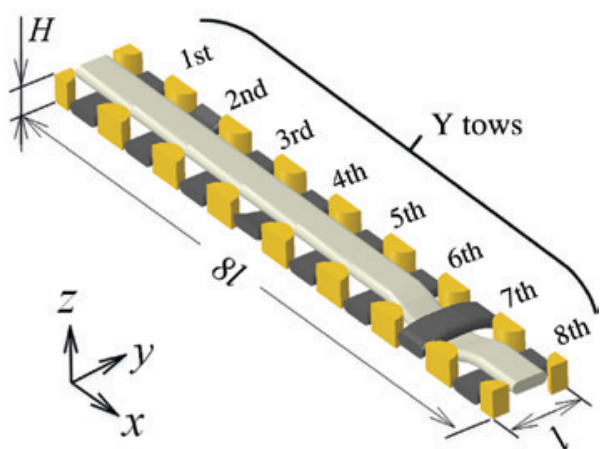

Figure 4: Periodic unit cell of weave composites.

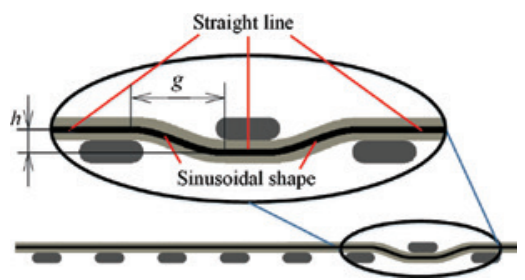

Figure 5: Central line of $\mathrm{X}$ tow: a combination of straight and sinusoidal shape lines.

an identical geometry the same as the $\mathrm{X}$ tow. It is consistent with the balance weave feature of the composites in this paper. In order to have a geometry of weave composites demonstrated and simulated conveniently, several assumptions are to be made based on micro-graphs of the material:

- The triaxial tows can be idealized as curved beams with a path depicted by straight lines and sinusoidal shape [defined in Equations (1)-(5) and shown in Figure 5].

- The cross section of typical X and Y tows can be idealized as "racetrack" shape, while the cross section of the $Z$ tows is idealized as a circle (shown in Figure 6).

- The tows are incompressible in transverse direction [23].

Dimensions of ORUC are measured in micro-graphs, and are listed in Table 1. The paths for the $\mathrm{X}$ and $\mathrm{Y}$ tows can be expressed as (where $z_{y}^{i}$ represents the path of the $i$-th Y tow):

$$
z_{x}= \begin{cases}-\frac{h}{2} \sin \frac{\pi(x-2 l)}{g} & x \in\left(2 l-\frac{g}{2}, 2 l+\frac{g}{2}\right) \\ \frac{h}{2} \sin \frac{\pi(x-3 l)}{g} & x \in\left(3 l-\frac{g}{2}, 3 l+\frac{g}{2}\right) \\ \frac{h}{2} & \text { Otherwise }\end{cases}
$$$$
z_{y}^{i}=-\frac{h}{2} \quad i=1,3,5,6,8
$$

A
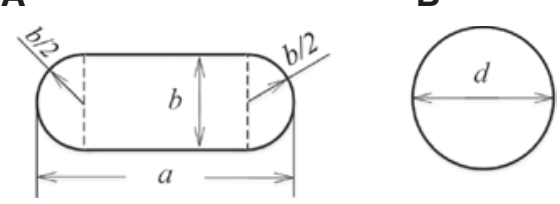

Figure 6: Cross section of tows in different directions.

(A) $\mathrm{X}$ and $\mathrm{Y}$ tows. (B) $\mathrm{Z}$ tows.

Table 1: Dimensions of ORUC (unit: $\mathrm{mm}$ ).

\begin{tabular}{lrlr}
\hline Dimensions & Value & Dimension & Value \\
\hline$a$ & 0.6 & $b$ & 0.2 \\
$d$ & 0.58 & $g$ & 0.9 \\
$h$ & 0.2 & $l$ & 1.3 \\
$H$ & 0.46 & - & - \\
\hline
\end{tabular}

$$
\begin{aligned}
& z_{y}^{2}= \begin{cases}-\frac{h}{2} & y \in\left[-\frac{l}{2}, \frac{l-g}{2}\right] \\
-\frac{h}{2} \sin \frac{\pi(2 y-l)}{2 g} & y \in\left(\frac{l-g}{2}, \frac{l}{2}\right]\end{cases} \\
& z_{y}^{4}= \begin{cases}-\frac{h}{2} \sin \frac{\pi(2 y+l)}{2 g} & y \in\left[-\frac{l}{2},-\frac{l-g}{2}\right) \\
\frac{h}{2} & y \in\left[-\frac{l-g}{2}, \frac{l}{2}\right]\end{cases} \\
& z_{y}^{6}= \begin{cases}\frac{h}{2} \sin \frac{\pi(2 y+l)}{2 g} & y \in\left[-\frac{l}{2},-\frac{l-g}{2}\right) \\
\frac{h}{2} & y \in\left[-\frac{l-g}{2}, \frac{l-g}{2}\right] \\
-\frac{h}{2} \sin \frac{\pi(2 y+l)}{2 g} & y \in\left(\frac{l-g}{2}, \frac{l}{2}\right]\end{cases}
\end{aligned}
$$

Based on these assumptions, the area and inertia moment of the $\mathrm{X}$ and $\mathrm{Y}$ tows are:

$$
\begin{gathered}
A_{f}=\frac{1}{4} \pi b^{2}+(a-b) b \\
I_{f}=\frac{1}{64} \pi b^{4}+\frac{1}{12}(a-b) b^{3}
\end{gathered}
$$

\subsection{Properties of constituent material}

It is assumed that the matrix zones are formed by mechanically isotropic amorphous carbon material [28]. Rao et al. 
[28] performed experiments to obtain the elastic properties of the carbon matrix. Based on these experiments, the Young's modulus and Poisson's ratio in this paper have been defined as $E_{m}=19 \mathrm{GPa}$ and $v_{m}=0.2$. The value of the shear modulus has been calculated using $G_{m}=E_{m} / 2\left(1+v_{m}\right)$ for isotropic materials.

The unidirectional fiber tow materials considered here is comprised of T300 carbon fibers, which are surrounded by amorphous carbon matrix to form tows. Considering their geometric features, fiber tows are treated as orthogonal anisotropic material [29]. Blacklock and Hayhurst [30] performed a finite element analysis of the tow to calculate the values for elastic and shear moduli and Poisson's ratios in the three principal directions. These values are listed in Table 2, and are employed in this paper to simulate the response of the composites.

\subsection{Mechanical model for woven fiber tows}

The mechanical behavior of 3D weave composites depends on the type of weave, fabric geometry, fiber volume fraction, and material constituents used. In the case of a uniaxial tension loading, the tows become straightened in the loading direction, while crimping and jamming occurs in the transverse direction. The matrix makes significant differences in supporting tows bearing tensile or compression loads, and tows in the $\mathrm{Y}$ and $\mathrm{Z}$ directions also enhance the tension and bending stiffness of the unit cell, which should be considered analyzing the elastic response. As the $Z$ tows are not undulated in the composites, suppose that the $Z$ tows do not interact with the $\mathrm{X}$ and $\mathrm{Y}$ tows directly.

In this paper, considering longitudinal tensile loading, the interaction between fiber tows and matrix was considered through two stages. First, the longitudinal tows are supported by matrix and tows in the transverse direction, and a homogenization process is adopted to simplify the mechanical analysis in the loading direction, as shown in Figure 7. Second, when stretched with longitudinal tows, the matrix shall shrink in the transverse direction due to Poisson's ratio, which forms a mechanical equilibrium between tows.

At the first stage, the mechanical support effect of the matrix and the $\mathrm{Y} / \mathrm{Z}$ tows can be idealized as a concentric beam stick to the axial fiber, herein called "mixed beam." The mixed beam has the same length as the $\mathrm{X}$ tow, with a hole along the centric line and containing several fiber tows vertical to the centric line ( $\mathrm{Y}$ and $\mathrm{Z}$ tows). Accounting for the geometry feature, equivalent elastic properties of the mixed beam can be calculated by the series-chain model (Reuss assumption [31]):
Table 2: Constituent material properties for $\mathrm{C} / \mathrm{C}$ composites.

\begin{tabular}{llll}
\hline Material & $\begin{array}{l}\text { Young's } \\
\text { modulus }\end{array}$ & $\begin{array}{l}\text { Poisson's } \\
\text { ratio }\end{array}$ & Shear modulus \\
\hline Unidirectional & $E_{f}=147.43 \mathrm{GPa}$ & $v_{f}=0.067$ & $G_{f}=18.04 \mathrm{GPa}$ \\
tows & $E_{f_{\perp}}=36.11 \mathrm{GPa}$ & $v_{f_{\perp}}=0.262$ & $G_{f_{\perp}}=16.03 \mathrm{GPa}$ \\
Amorphous & $E_{m}=19.00 \mathrm{GPa}$ & $v_{m}=0.2$ & $G_{m}=7.95 \mathrm{GPa}$ \\
carbon matrix & & & \\
\hline
\end{tabular}

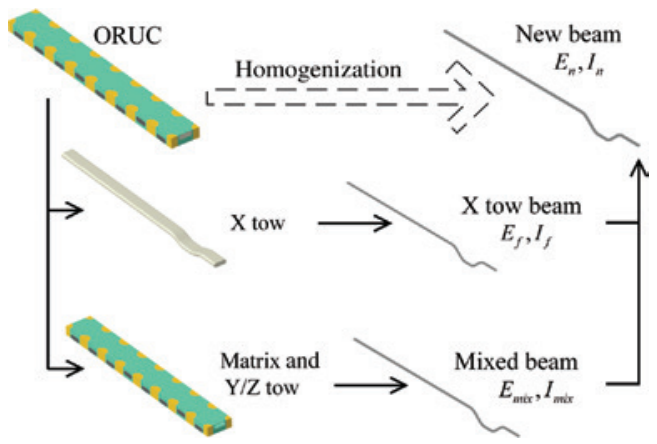

Figure 7: Simplification of tows and matrix support in longitudinal tensile loading.

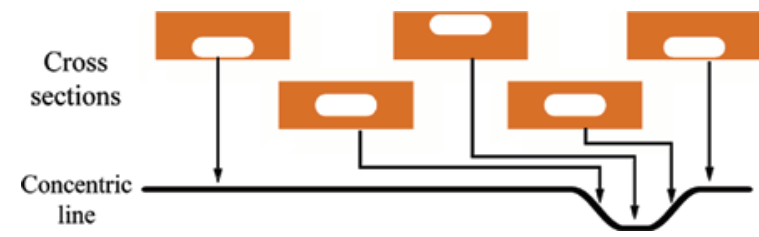

Figure 8: Cross sections of mixed beam.

$$
E_{\text {mix }}=\frac{\left(1-c_{x}\right) E_{f_{\perp}} E_{m}}{c_{m} E_{f_{\perp}}+\left(c_{y}+c_{z}\right) E_{m}}
$$

where $c_{m}, c_{x}, c_{y}$, and $c_{z}$ represent the volume fraction of the matrix and fiber tows in three directions, respectively. The cross sections of the mixed beam vary in different positions due to the undulation of the $\mathrm{X}$ tow. Thus, its inertia moment $I_{\text {mix }}$ is a continuous variable along the axial direction, as demonstrated in Figure 8, while its area $A_{\text {mix }}$ is a constant. For simplification, the average value of $I_{\text {mix }}=0.01681 \mathrm{~mm}^{4}$ will be employed in the following simulation.

Suppose the interface between the matrix and fiber tows are perfect, the displacement of the $\mathrm{X}$ tow and mixed beam would be identical; therefore, they could be treated as a single beam with new properties. Suppose the elastic modulus, inertia moment, and section area are $E_{n}, I_{n}$, and $A_{n}$, respectively, these parameters should satisfying: 


$$
\left\{\begin{array}{l}
E_{n} I_{n}=E_{f} I_{f}+E_{\text {mix }} I_{\text {mix }} \\
E_{n} A_{n}=E_{f} A_{f}+E_{\text {mix }} A_{\text {mix }}
\end{array}\right.
$$

In order to simplify the geometrical description, new beams (with properties of $E_{n}, I_{n}$, and $A_{n}$ ) will still be named $\mathrm{X}$ tows. Elastic properties of $\mathrm{Y}$ tows could be revised using the same method.

Figure 9A illustrates the interaction between biaxial tows. In the interlacing part of the cell, there should be an interaction force only if at least one interlacing tow is curved. Thus, there is no interaction force in the interlaced 1st, 3rd, and 5th Y tows. According to the balance weave feature and periodicity of ORUC, interlacing forces 2, 4, 6, and 8 are equivalent. They shall share a single semi-bending interaction force. Assume the semi-bending and bending interaction forces being $N_{a}, N_{b}$; likewise, the longitudinal tensile force and constraint moment being $N_{1}, M_{1}$, and transversal constraint moment being $M_{2}$, respectively, as indicated in Figure 9. It should be noted that eight $Y$ tows are linked together in sequences as aforementioned; thus, their external load can be represented by $M_{2}$, and the internal loads between Y tows (linked by a dashed line in Figure 9B) will be derived later.

Based on the assumptions of the tow geometry and load conditions stated above, the moments of $\mathrm{X}$ tow can be deduced:

$$
M_{x}=\left\{\begin{array}{l}
M_{1} \\
M_{1}+\frac{5}{2} N_{a} l+N_{a} \chi \\
M_{1}+3 N_{a} l+2 N_{a} \chi \\
M_{1}+\frac{3}{2} N_{a} l+3 N_{a} \chi \\
M_{1}+\frac{3}{2} N_{a} l+3 N_{a} x-\frac{1}{2} N_{1} h\left(\sin \frac{\pi(x-2 l)}{g}+1\right) \\
M_{1}+\frac{3}{2} N_{a} l+3 N_{a} x-N_{1} h \\
M_{1}+\frac{3}{2} N_{a} l+3 N_{a} x-N_{1} h+N_{b}\left(\frac{5 l}{2}-x\right) \\
M_{1}+\frac{3}{2} N_{a} l+3 N_{a} x+N_{a}\left(\frac{3 l}{2}+3 x\right)+\frac{1}{2} N_{1} h(\sin \\
M_{1}+\frac{3}{2} N_{a} l+3 N_{a} x+N_{b}\left(\frac{5 l}{2}-x\right) \\
M_{1}-2 N_{a} l+5 N_{a} x+N_{b}\left(\frac{5 l}{2}-x\right)
\end{array}\right.
$$$$
\begin{aligned}
& x \in\left[-4 l,-\frac{5 l}{2}\right) \\
& x \in\left[-\frac{5 l}{2},-\frac{l}{2}\right) \\
& x \in\left[-\frac{l}{2}, \frac{3 l}{2}\right) \\
& x \in\left[\frac{3 l}{2}, 2 l-\frac{g}{2}\right) \\
& x \in\left[2 l-\frac{g}{2}, 2 l+\frac{g}{2}\right) \\
& x \in\left[2 l+\frac{g}{2}, \frac{5 l}{2}\right) \\
& x \in\left[\frac{5 l}{2}, 3 l-\frac{g}{2}\right) \\
& x \in\left[3 l-\frac{g}{2}, 3 l+\frac{g}{2}\right) \\
& x \in\left[3 l+\frac{g}{2}, \frac{7 l}{2}\right) \\
& x \in\left[\frac{7 l}{2}, 4 l\right]
\end{aligned}
$$

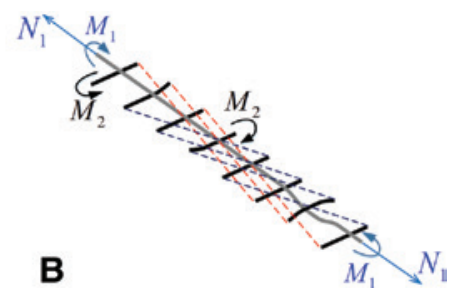

Figure 9: External load and interaction force of $X$ and $Y$ tows. (A) Interaction force between $X$ and $Y$ tows. (B) External load of $X$ and Y tows.

Meanwhile, the moments of the Y tows are:

$$
\begin{gathered}
M_{y}^{1}=M_{2} \\
M_{y}^{6}= \begin{cases}M_{2} & y \in\left[-\frac{l}{2}, 0\right) \\
M_{2}+N_{a} y & y \in\left[0, \frac{l}{2}\right]\end{cases} \\
M_{y}^{8}= \begin{cases}M_{2}^{3}=M_{2}+N_{a}(y+l) \\
M_{2}+N_{a}(2 y+2 l) & y \in\left[0, \frac{l}{2}\right]\end{cases}
\end{gathered}
$$




$$
\begin{gathered}
M_{y}^{5}=M_{2}+N_{a}(2 y+4 l) \\
M_{y}^{2}= \begin{cases}M_{2}+N_{a}(2 y+6 l) & y \in\left[-\frac{l}{2}, 0\right) \\
M_{2}+N_{a}(3 y+6 l) & y \in\left[0, \frac{l}{2}\right]\end{cases} \\
M_{y}^{7}= \begin{cases}M_{2}+N_{a}(3 y+9 l) & y \in\left[-\frac{l-g}{2}, 0\right) \\
M_{2}+N_{a}(3 y+9 l)-N_{b} y & y \in\left[0, \frac{l}{2}\right]\end{cases} \\
M_{y}^{4}= \begin{cases}M_{2}+N_{a}(3 y+12 l)-N_{b}(y+l) & y \in\left[-\frac{l}{2}, 0\right) \\
M_{2}+N_{a}(4 y+12 l)-N_{b}(y+l) & y \in\left[0, \frac{l}{2}\right]\end{cases}
\end{gathered}
$$

where $M_{y}^{i}$ represents the moment of the $i$-th $\mathrm{Y}$ tow in the unit cell. The on-axial force of the $\mathrm{X}$ tow is:

$$
N_{x}= \begin{cases}\frac{N_{1}}{\sqrt{1+\frac{h^{2} \pi^{2}}{4 g^{2}} \cos ^{2} \frac{\pi(x-2 l)}{g}}} & x \in\left(2 l-\frac{g}{2}, 2 l+\frac{g}{2}\right) \\ \frac{N_{1}}{\sqrt{1+\frac{h^{2} \pi^{2}}{4 g^{2}} \cos ^{2} \frac{\pi(x-3 l)}{g}}} & x \in\left(3 l-\frac{g}{2}, 3 l+\frac{g}{2}\right) \\ N_{1} & \text { Otherwise }\end{cases}
$$

From Equations (10) to (19), the complementary strain energy of the $\mathrm{X}$ tow in the unit cell is:

$$
U_{x}=\frac{1}{2 E_{n} I_{n}} \int_{-4 l}^{4 l} M_{x}^{2} d l+\frac{1}{2 E_{n} A_{n}} \int_{-4 l}^{4 l} N_{x}^{2} d l
$$

The complementary strain energy of the $\mathrm{Y}$ tows in the unit cell can be obtained in a similar way:

$$
U_{y}=\sum_{i=1}^{8}\left(\frac{1}{2 E_{n} I_{n}} \int_{-l / 2}^{l / 2}\left(M_{y}^{i}\right)^{2} d l\right)
$$

Therefore, the total complementary energy of the RUC can be obtained as:

$$
\Pi=U_{x}+U_{y}
$$

As on-axial forces and moments are liner combinations of external and interaction loads, the total complementary energy could be deduced to a quadratic form. Defining $D_{1}=N_{1}, D_{2}=M_{1}, D_{3}=M_{2}, D_{4}=N_{a}, D_{5}=N_{b}$, then:

$$
\Pi=\sum_{i=1}^{5} \sum_{j=1}^{5} k_{i j} D_{i} D_{j}
$$

where the coefficients $k_{i j}$ are constants concerning geometric parameter, material properties, and values $J_{0}$ to $J_{4}$. $J_{0}$ to $J_{4}$ are defined as:

$$
\begin{aligned}
& J_{0}=\int_{0}^{g / 2} \sqrt{1+\frac{h^{2} \pi^{2}}{4 g^{2}} \cos ^{2} \frac{\pi x}{g}} d x \\
& J_{1}=\int_{0}^{g / 2} \frac{1}{\sqrt{1+\frac{h^{2} \pi^{2}}{4 g^{2}} \cos ^{2} \frac{\pi x}{g}}} d x \\
& J_{2}=\int_{0}^{g / 2} x^{2} \sqrt{1+\frac{h^{2} \pi^{2}}{4 g^{2}} \cos ^{2} \frac{\pi x}{g}} d x
\end{aligned}
$$

$$
\begin{aligned}
& J_{3}=\int_{0}^{g / 2} \sin ^{2}\left(\frac{\pi x}{g}\right) \sqrt{1+\frac{h^{2} \pi^{2}}{4 g^{2}} \cos ^{2} \frac{\pi x}{g}} d x \\
& J_{4}=\int_{0}^{g / 2} x \sin \left(\frac{\pi x}{g}\right) \sqrt{1+\frac{h^{2} \pi^{2}}{4 g^{2}} \cos ^{2} \frac{\pi x}{g}} d x
\end{aligned}
$$

According to the complementary variation principle, the system should be stable in the minimized total complementary energy, which should lead to:

$$
\frac{\partial \Pi}{\partial D_{i}}=0 \quad i=2,3,4,5
$$

Equation (29) forms a set of liner equations of $D_{i}$. The results obtained by solving the equations are:

$$
D_{i}=\eta_{i} N_{1} \quad i=2,3,4,5
$$

where $\eta_{i}$ are constants, which indicates the forces and moments are liner forms of $N_{1}$.

Based on the potential energy principle, deformation of the unit cell in the $\mathrm{X}$ direction under external and internal load could be derived:

$$
\delta_{x}=\int_{-4 l}^{4 l} \frac{M_{x}}{E_{n} I_{n}} \frac{\partial M_{x}}{\partial N_{1}} d l+\int_{-4 l}^{4 l} \frac{N_{x}}{E_{n} A_{n}} \frac{\partial N_{x}}{\partial N_{1}} d l
$$

Therefore, the elastic modulus of the woven tows in the $\mathrm{X}$ direction could be deduced as:

$$
E_{x t}=\frac{\overline{\sigma_{x x}}}{\overline{\varepsilon_{x x}}}=\frac{8 N_{1}}{H \delta_{x}}
$$



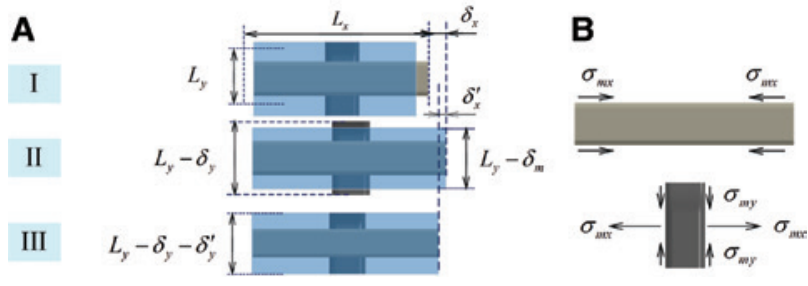

Figure 10: Steps to demonstrate the effect of Poisson's ratio of matrix; only one $Y$ tow is shown for easy reading.

(A) Three steps. (B) Stress applied to tows from matrix.

In the second stage, the effect of the Poisson's ratio of the matrix is considered. Three steps are taken to carry out the process. Figure 10 illustrate the steps in the X-Y plane. Step I, ORUC is not deformed, $L_{x}$ and $L_{y}$ are the initial dimensions. Step II, regardless of the interaction between matrix and transverse tows, when longitudinal tow stretches $\delta_{x}$, the stress of the matrix is $\sigma_{0}=\frac{\delta_{x} E_{m}}{L_{x}}$. The matrix shall shrink $\delta_{m y}$ and $\delta_{m z}$ in the $\mathrm{Y}$ and $\mathrm{Z}$ directions:

$$
\delta_{m y}=\frac{\delta_{x} v_{m} L_{y}}{L_{x}}, \delta_{m z}=\frac{\delta_{x} v_{m} L_{z}}{L_{x}}
$$

The shift of the $\mathrm{Y}$ and $\mathrm{Z}$ tows in their axial direction due to Poisson's ratio $v_{f}$ are:

$$
\delta_{y}=\frac{\sigma_{0}}{E_{f_{\perp}}} v_{f} L_{y}, \delta_{z}=\frac{\sigma_{0}}{E_{f_{\perp}}} v_{f} L_{z}
$$

Step III, equalize the deformation of the matrix and transverse tows. The stress increment of the matrix from step II to III in different directions are:

$$
\sigma_{m x}=\frac{\delta_{x}^{\prime}}{L_{x}} E_{x t}, \sigma_{m y}=\frac{\delta_{y}^{\prime}}{L_{y}} E_{y t}, \sigma_{m x}=\frac{\delta_{z}^{\prime}}{L_{z}} E_{z t}
$$

where $E_{y t}=E_{x t}$, and $E_{z t}$ is calculated as the same method with the homogenization process of the mixed beam:

$$
E_{z t}=c_{z} E_{f}+\frac{\left(1-c_{z}\right)^{2} E_{f_{\perp}} E_{m}}{\left(c_{x}+c_{y}\right) E_{m}+c_{m} E_{f_{\perp}}}
$$

The strain increment of the matrix from step II to III could also be deduced:

$$
\varepsilon_{x}=-\frac{\delta_{x}^{\prime}}{L_{x}}, \varepsilon_{y}=\frac{\delta_{m y}-\delta_{y}-\delta_{y}^{\prime}}{L_{y}}, \varepsilon_{x}=\frac{\delta_{m z}-\delta_{z}-\delta_{z}^{\prime}}{L_{z}}
$$

Equations (35) and (37) could be substituted into constitutive equations of the matrix:

$$
\left(\begin{array}{l}
\varepsilon_{x} \\
\varepsilon_{y} \\
\varepsilon_{z}
\end{array}\right)=\left(\begin{array}{ccc}
\frac{1}{E_{m}} & -\frac{v_{m}}{E_{m}} & -\frac{v_{m}}{E_{m}} \\
-\frac{v_{m}}{E_{m}} & \frac{1}{E_{m}} & -\frac{v_{m}}{E_{m}} \\
-\frac{v_{m}}{E_{m}} & -\frac{v_{m}}{E_{m}} & \frac{1}{E_{m}}
\end{array}\right)\left(\begin{array}{l}
\sigma_{x} \\
\sigma_{y} \\
\sigma_{z}
\end{array}\right)
$$

By solving equation (38), $\delta_{x}^{\prime}, \delta_{y}^{\prime}$, and $\delta_{z}^{\prime}$ could be obtained. The elastic modulus and Poisson's ratio of weave composites are

$$
\left\{\begin{array}{l}
E_{x x}=\frac{8 N_{1}}{H\left(\delta_{x}-\delta_{x}^{\prime}\right)} \\
v_{x y}=\frac{\left(\delta_{y}+\delta_{y}^{\prime}\right) L_{x}}{\left(\delta_{x}-\delta_{x}^{\prime}\right) L_{y}} \\
v_{x z}=\frac{\left(\delta_{z}+\delta_{z}^{\prime}\right) L_{x}}{\left(\delta_{x}-\delta_{x}^{\prime}\right) L_{z}}
\end{array}\right.
$$

When applying uniaxial loading in the $\mathrm{Z}$ direction, assume that the deformation of the $\mathrm{Z}$ tow is $\delta_{z t^{t}}$. For the limitation of the chapter, a detailed deviation process of the gas has been saved; the elastic moduli $E_{z z}$ could be deduced in a similar manner:

$$
E_{z z}=\frac{\delta_{z t}+\delta_{z t}^{\prime \prime}}{\delta_{z}} E_{z f}
$$

Hence, the elastic properties except shear moduli are derived.

The analytical curved beam model proposed in this paper considered the interacting force between the $\mathrm{X}$ and $Y$ tows as well as the supporting function and Poisson's ratio of matrix, which derives the elastic properties of the composites directly. The verification and comparisons are made in the following sections.

\section{Finite element model of representative unit cell}

In order to verify the validation of the analytical model, a finite element method (FEM) model based on periodic boundary conditions is established to predict the engineering constants of $\mathrm{C} / \mathrm{Cs}$.

\subsection{Finite element model}

An FEM model is established using the commercial analysis software ABAQUS (Dassault Systèmes Simulia Corp., USA), as shown in Figure 11. The $\mathrm{X}, \mathrm{Y}$, and $\mathrm{Z}$ tows 


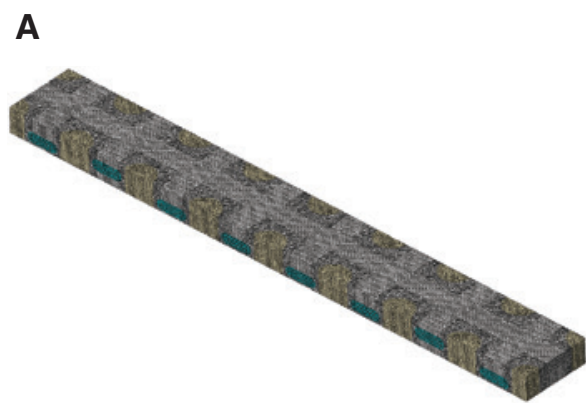

Figure 11: FEM model of ORUC.

(A) Elements of the whole model. (B) Elements of fiber tows.

and matrix, which have the same geometrical characters described in Section 2.1, make up the entire ORUC. As failure is not considered in this model, the interface between tows and matrix is assumed to be perfect; thus, the tows and matrix are constructed within a single part, sharing one set of nodes on the interface to simulate the fully contacted interface. The mesh for the woven tows consists of 120032 C3D8 (eight-node liner brick) and 3232 C3D6 (six-node liner triangular prism) elements, and the mesh for the matrix consists of 34944 C3D8, 2688 C3D6, and 150704 C3D4 (four-node liner tetrahedron) elements.

\subsection{Periodic boundary conditions}

A periodic boundary conditions is essential in RUC model [14, 32]. Xia et al. [33] proposed an explicit unified form of periodic boundary conditions for a parallelepiped-shaped periodic RUC model, which proved satisfying not only for the boundary displacement periodicity but also for the boundary traction periodicity. For parallelepiped RUC models, a unified periodic boundary conditions practically in commercial finite analysis software is obtained:

$$
u_{i}^{j+}-u_{i}^{j-}=c_{i}^{j}
$$

where $u_{i}^{j+}$ and $u_{i}^{j-}$ represent a pair of nodes in parallel boundary faces.

The boundary of the ORUC model in this paper is split into eight faces as shown in Figure 12. Thus, the boundary condition can be derived from equation (41) as:

$$
\left\{\begin{array}{l}
u_{a b c d}-u_{e f g h}=c_{1} \\
u_{f g c b}-u_{e h d a}=c_{2} \\
u_{n m c g}-u_{\text {eapq }}=c_{3} \\
u_{n h d m}-u_{f q p b}=c_{4}
\end{array}\right.
$$

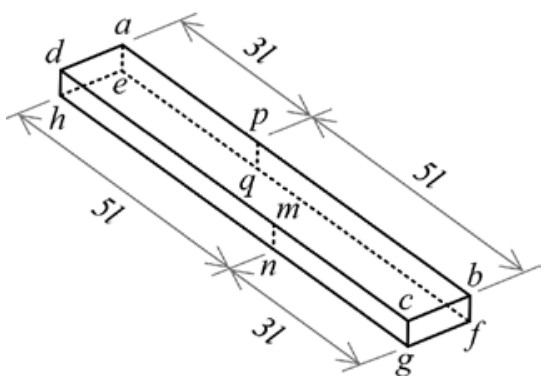

Figure 12: Faces split from boundary in periodic boundary conditions.

where $u_{a b c d}$ represents the displacement of each node on the boundary surface $S_{a b c d}$, etc.

In this paper, a master-slavery-nodes method is employed, where the nodes $a, e, f$, and $n$ are master nodes, other nodes in the boundary are slavery nodes, as shown in Figure 12. The external load on the ORUC can be applied by determining the displacement of the master nodes [33]. According to equation (42), each constant $c_{i}$ could be represented by the displacement of a pair of corresponding nodes. Thus, there are some equations available: $c_{1}=u_{a}-u_{e}, c_{2}=u_{f}-u_{e}, c_{3}=u_{n}-u_{e}, c_{4}=u_{n}-u_{f}$, Where $u_{a}$ represents the displacement of the node located in point $a$, etc. Equation (42) can derive into the following equations:

$$
\left\{\begin{array}{l}
u_{a b c d}-u_{e f g h}=u_{a}-u_{e} \\
u_{f g c b}-u_{e h d a}=u_{f}-u_{e} \\
u_{n m c g}-u_{e a p q}=u_{n}-u_{e} \\
u_{n h d m}-u_{f q p b}=u_{n}-u_{f}
\end{array}\right.
$$

It should be noted that faces $S_{a b f e}$ and $S_{b c g h}$ are each split into two faces, and periodic boundary conditions corresponds diagonally.

Equation (43) is used to set constraints to the FEM model between each pair of nodes at parallel surfaces via the Python script in ABAQUS. It should be mentioned that constraints in the edges and vertexes may be over 
Table 3: Load methods and corresponding master node displacement.

\begin{tabular}{llll}
\hline Load methods & \multicolumn{2}{l}{ Displacement of nodes } \\
\cline { 2 - 4 } & $\boldsymbol{a}$ & $\boldsymbol{f}$ & $\boldsymbol{n}$ \\
$\overline{\overline{\sigma_{x x}}}$ & $(0,0,-)$ & $\left(8 \overline{\varepsilon_{x x}} l, 0,0\right)$ & $(-,-, 0)$ \\
$\overline{\sigma_{y y}}$ & $(0,0,-)$ & $(-, 0,0)$ & $\left(-, \overline{\varepsilon_{x x}} l, 0\right)$ \\
$\overline{\sigma_{z z}}$ & $\left(0,0, \overline{\varepsilon_{z z}} l\right)$ & $(-, 0,0)$ & $(-,-, 0)$ \\
$\overline{\sigma_{x y}}$ & $(0,0,0)$ & $(0,0,0)$ & $\left(\overline{\varepsilon_{x y} l}, 0,0\right)$ \\
$\overline{\sigma_{y z}}$ & $\left(0, \overline{\varepsilon_{y z}} h, 0\right)$ & $(0,0,0)$ & $(0,0,0)$ \\
$\overline{\sigma_{z x}}$ & $\left(\overline{\varepsilon_{z x}} h, 0,0\right)$ & $(0,0,0)$ & $(0,0,0)$ \\
\hline
\end{tabular}

constrained due to their situation in several faces, and the minimum number of combinations of constraints needs to be deduced to avoid an over constrained degree of freedom.

\subsection{Load method and elastic properties of ORUC}

Elastic properties are obtained by measuring the displacement or reaction force of master nodes in different load methods. Each type of load method, corresponding to a component of stress tensor $\left(\overline{\sigma_{i j}}\right)$, is applied to the FEM model by assigning a displacement of master nodes. Through measuring displacements and reaction forces, the mean stress and strain could be deduced and then used to obtain elastic constants. The displacements of the master nodes in the load methods are given in Table 3. Values in parentheses mean displacements in the $\mathrm{X} / \mathrm{Y} / \mathrm{Z}$ directions, respectively. It should be mentioned that the displacement "_" means no constraint in the corresponding degree of freedom, and their displacements ought to be measured after the numerical computation.

The computation process is performed in the software ABAQUS/Standard. In the case of unidirectional tensile loading $\sigma_{x x}$, the stress and displacement nephograms of ORUC are shown in Figure 13.

As demonstrated in Ref. [33], the average stress and $\operatorname{strain}\left(\overline{\sigma_{x x}}\right.$ and $\overline{\varepsilon_{x x}}$ ) of the ORUC can be calculated by displacements and reaction forces of master nodes. Regarding dimensions of the ORUC, $\overline{\sigma_{x x}}$ and $\overline{\varepsilon_{x x}}$ are:

$$
\overline{\sigma_{x x}}=\frac{P_{x}^{f}}{l H}, \overline{\varepsilon_{x x}}=\frac{\Delta_{x}^{f}}{8 l}
$$

where $P_{x}^{f}$ and $\Delta_{x}^{f}$ are the reaction force and displacement of the node $f$ in the $\mathrm{X}$ direction. Elastic tensile modulus and Poisson's ratio can be calculated by:

$$
E_{x}=\frac{\overline{\sigma_{x}}}{\varepsilon_{x}}, v_{x y}=-\frac{8 \Delta_{y}^{n}}{\Delta_{x}^{f}}, v_{x z}=-\frac{8 l \Delta_{z}^{n}}{H \Delta_{x}^{f}},
$$

Other elastic constants can be deduced in a similar manner. Predictions of elastic constants in the FEM model are listed in Equation (46).

$$
\left\{\begin{array}{l}
E_{x x}=47.606 \mathrm{GPa}, E_{y y}=47.436 \mathrm{GPa}, E_{z z}=44.827 \mathrm{GPa} \\
G_{x y}=12.135 \mathrm{GPa}, G_{y z}=11.702 \mathrm{GPa}, G_{z x}=11.675 \mathrm{GPa} \\
v_{x y}=0.100, v_{y z}=0.111, v_{z x}=0.111
\end{array}\right.
$$

\subsection{Convergence study for mesh size}

A mesh size convergence study has been conducted for the ORUC model. Three different mesh sizes have been tested: $0.1 \mathrm{~mm}, 0.04 \mathrm{~mm}$, and $0.02 \mathrm{~mm}$. The model has 31,311 elements if the mesh size is set to $0.1,311,600$ elements for
A

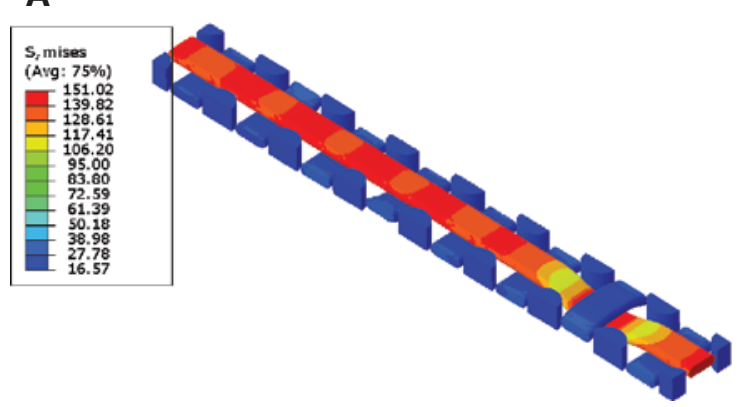

B

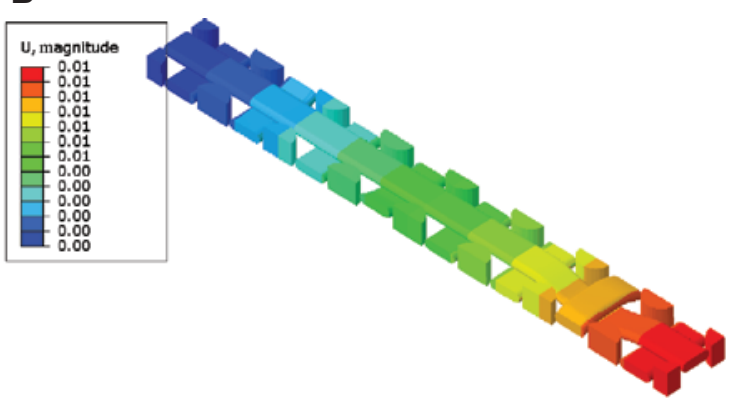

Figure 13: Stress and displacement nephograms of ORUC under unidirectional tensile loading $\varepsilon_{x x^{*}}$ (A) Mises stress nephogram. (B) Magnitude displacement nephogram. 
Table 4: Convergence study for mesh size.

\begin{tabular}{lrlrrr}
\hline Mesh size & Elements & & $\boldsymbol{E}_{x x}$ & $\boldsymbol{E}_{z z}$ & $\boldsymbol{\sigma}_{\text {mises }}^{\max }$ \\
\hline $0.1 \mathrm{~mm}$ & 31,311 & Value & 47.108 & 44.527 & 148.21 \\
$0.04 \mathrm{~mm}$ & 311,600 & Value & 47.606 & 44.828 & 155.87 \\
& & Diff & $1.06 \%$ & $0.68 \%$ & $5.17 \%$ \\
\multirow{2}{*}{$0.02 \mathrm{~mm}$} & \multirow{2}{*}{$2,148,181$} & Value & 44.730 & 44.905 & 158.76 \\
& & Diff & $0.26 \%$ & $0.17 \%$ & $1.85 \%$ \\
\hline
\end{tabular}

mesh size 0.04, and 2,148,181 elements for mesh size 0.02 . The results in Table 4 show that for mesh sizes 0.04-0.02, the difference in the predicted moduli and maximum mises stress is less than $0.3 \%$ and $2 \%$. However, the computational time for the mesh size 0.02 model to the mesh size 0.04 model is more than doubled. Therefore, the mesh size of the ORUC model has been assigned to 0.04 .

\section{Experiment validation}

\subsection{Experiment configuration}

As there are few experimental results for the 3D eight-harness satin weave composites in the previous literatures, experiment tests were performed to evaluate the mechanical properties of the material. The specimens are manufactured from the full thickness sheet composites and piercing fiber tows (Beijing North XinYuan Electrical Carbon Products Co., Beijing, China). The carbon matrix, which surrounds the fibers, is manufactured by the CVI technique [34]. The phenolic resin composite is first carbonized at temperatures up to $2500^{\circ} \mathrm{C}$ to create a porous structure. It then undergoes a CVI process in which a hydrocarbon gas is "cracked" at about $1000^{\circ} \mathrm{C}$. The resulting carbon is deposited in the porous carbon fiber structure.

The specimen shape is given in Figure 14, and dimensions $h$ and $w$ are listed in Table 5. The radius, R14.5, was introduced to ensure that there would not be any failure appearing in the side of the specimen first. The specimens were tested in an INSTRON 250KN tensile testing machine (8802; Instron Corp., USA). As there is no well-recognized testing standard, the tests were carried out under a constant displacement rate of $0.5 \mathrm{~mm} / \mathrm{min}$, which should be at an equivalent strain rate of $3 \times 10^{-5} \mathrm{~s}^{-1}$. The determination of this value was discussed by Sheikh et al. [35].

As shown in Figure 14B, each specimen was instrumented with four electrical resistance strain gauges (front and back). Their purpose was to enable axial alignment of the specimen at low loads before the test was started

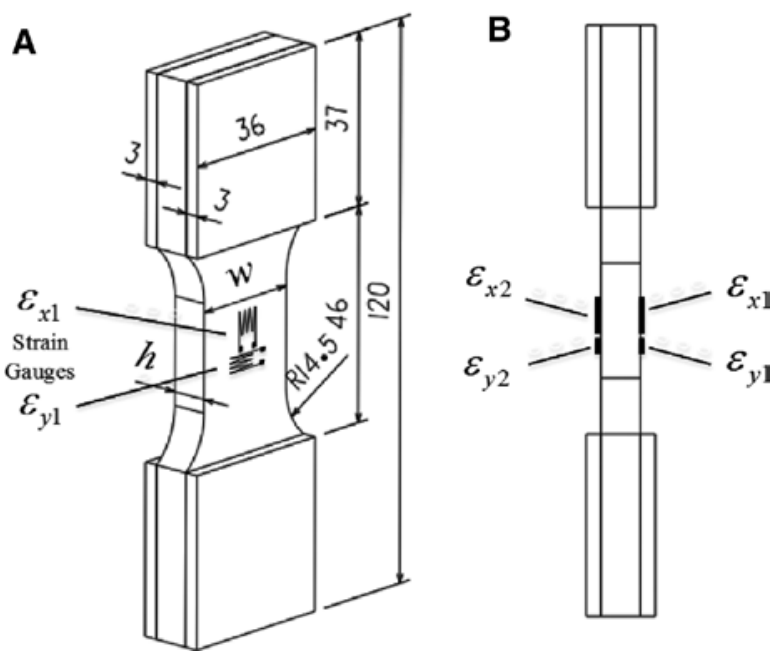

Figure 14: Geometry and strain gauges of specimen. (A) Geometry. (B) Strain gauges.

Table 5: Dimensions and test results of specimen.

\begin{tabular}{lrrrrrr}
\hline Specimens & \multicolumn{2}{c}{ Dimensions $(\mathrm{mm})$} & & $\begin{array}{r}\boldsymbol{E}_{\boldsymbol{x}} \\
(\mathrm{GPa})\end{array}$ & $\boldsymbol{v}_{x y}$ & $\begin{array}{r}\boldsymbol{\sigma}_{b} \\
(\mathrm{MPa})\end{array}$ \\
\cline { 2 - 5 } & $\boldsymbol{h}$ & $\boldsymbol{w}$ & & & \\
\hline $\mathrm{T}-1$ & 8.035 & 24.845 & 47.905 & 0.1042 & 168.92 \\
$\mathrm{~T}-2$ & 8.050 & 24.855 & 46.263 & 0.1165 & 172.27 \\
Average & 8.043 & 24.850 & 47.084 & 0.1104 & 170.59 \\
\hline
\end{tabular}

and to provide a means of measuring the strain throughout the test. Considering the extremely high material cost, only two specimens were carefully prepared and tested. The loading speed was controlled at $0.5 \mathrm{~mm} / \mathrm{min}$ until the ultimate failure of the structures. The axial load, displacement, and stain were measured during the test. All the results will be given in the following section.

\subsection{Experiment results}

The experimental results are listed in Table 5. As seen in the stress-strain curves in Figure 15, two specimens are in excellent repeatability. There is an apparent liner relationship between the stress and strain before half of the ultimate load. Therefore, tensile elastic properties of the specimen are calculated from the stress-strain slope of fitting within this region.

\section{Comparisons and discussions}

To validate the analytical and FEM models in this paper, comparisons have been made between predictions and 


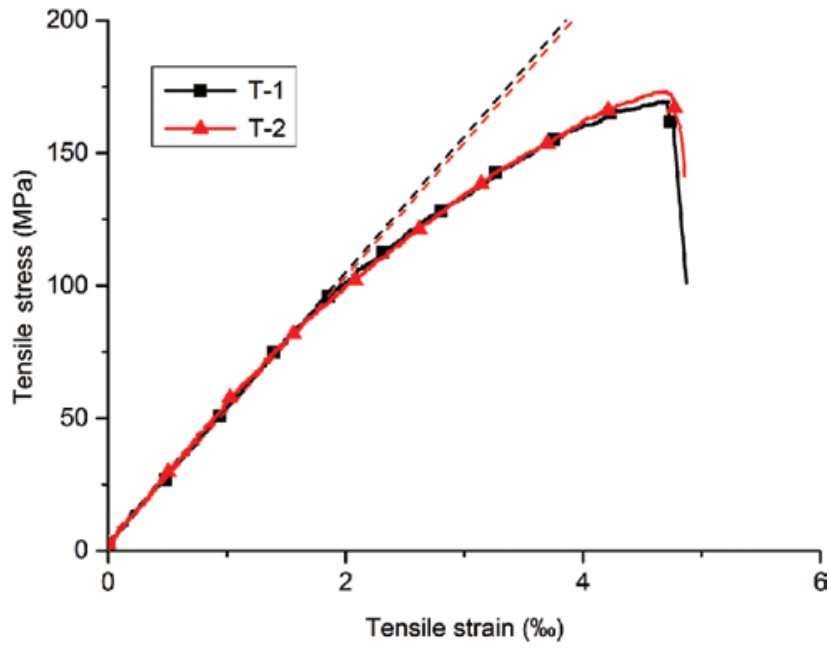

Figure 15: Stress-strain data of experiment.

experiments, as well as predictions of several models in literatures. The sensitivity effects of several parameters are also discussed.

\subsection{Elastic property}

Several models are employed to predict the tensile modulus of the composites, which are the bridging model [18] and the iso-stress and iso-strain model [17]. The experimental results are tabled in Table 6 along with the predictions of the different models. It could easily be found that the predictions of the FEM model show the minimum errors for all the experiment results. Among the analytical models, the curved beam model agree well with the experimental results, which indicates the validity of the curved beam model addressed in this paper.

Table 6 indicates that the moduli predictions of the curved beam model, FEM model, and bridging model are between the predictions of the iso-tress model and isostrain model. This result is consistent with the conclusion of the previous work [17] that the iso-strain model gives an upper bound of the prediction for fabric composites, and the iso-stress model gives a lower bound.

From the previous work, it could be concluded that all the iso-strain/stress model and bridging model are based on the classical laminated theory, which ignore the interaction of interlacing strands. Some models in the literature [22-24] take interacting force into consideration, yet the effect of the matrix was ignored in the mechanical process of strands and are not suitable for satin weave composites. However, the curved beam model in this paper considered the interlacing force between the orthogonal strands and the effects of the matrix supporting and Poisson's ratio, along with the undulation and practical cross section of the strands and actual representative boundary conditions, to predict the elastic properties of satin weave composites. The relative error of the predicted tensile modulus is less than $2 \%$ compared to the experimental results, which is quite good for an analytical model.

\subsection{Sensitivity study}

A sensitivity study is conducted for the variation of the constitutive elastic properties. By comparing the predictions of the different models, the validity and stability can be suggested.

\subsubsection{The constitutive elastic properties}

A wide range of constitutive elastic properties is set in different models to predict the elastic moduli. The results are show in Figures 16 and 17

. The iso-stress model is excluded due to its huge deviation. As the FEM model is the most accurate one, all the predictions from the analytical models will be compared with those from the FEM model for each variation parameter.

From Figure 16, it can be concluded that while all the models show positive correlation between the predicted

Table 6: Comparison between experiments and predictions in different models.

\begin{tabular}{lrrrr}
\hline Model type & $\boldsymbol{E}_{x x}$ & Relative deviation & $\boldsymbol{v}_{x y}$ & Relative deviation \\
\hline Experiment & 47.084 & - & 0.1104 & - \\
FEM model & 47.606 & $1.11 \%$ & 0.1000 & $9.42 \%$ \\
Curved beam model & 46.383 & $1.49 \%$ & 0.0979 & $-11.32 \%$ \\
Bridging model & 45.342 & $3.70 \%$ & - & - \\
Iso-strain model & 49.533 & $5.20 \%$ & - & - \\
Iso-stress model & 27.905 & $40.73 \%$ & - & - \\
\hline
\end{tabular}



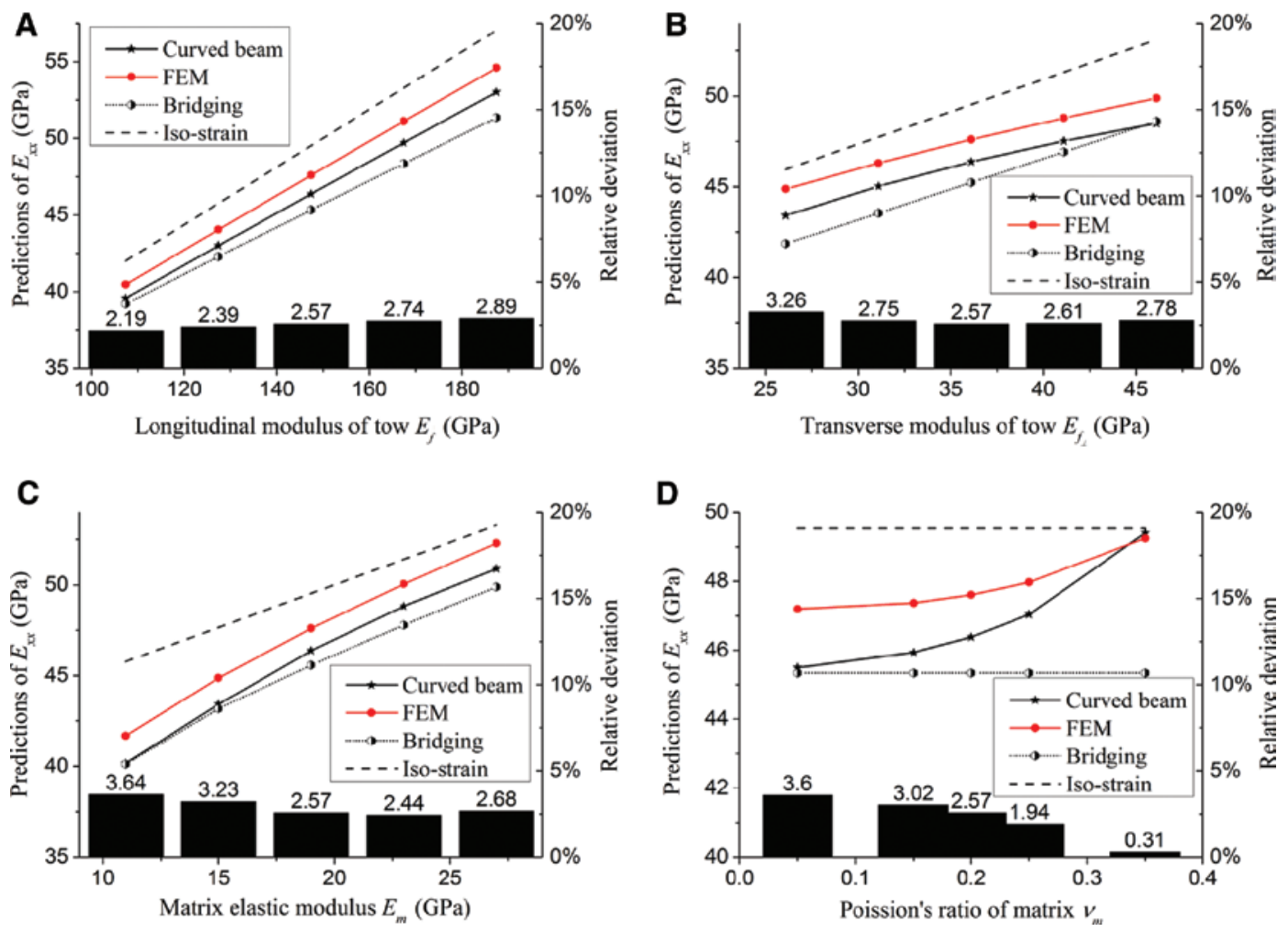

Figure 16: The predicted $E_{x x}$ (lines with left axis) and relative deviation of curved beam model (column bars with right axis) from different models with variant constitutive properties

(A) Longitudinal modulus of tow $E_{f}$ (B) Transverse modulus of tow $E_{f_{\perp}}$. (C) Matrix elastic modulus $E_{m}$. (D) Poisson's ratio of matrix $v_{m}$.

moduli and the constitutive properties, they manifest different relative deviations. The predictions of the iso-strain model still show the upper bound of all the other models, which corresponds to the results in the literature [17].

Comparing to the predictions from the FEM model, the relative deviation of the curved beam model is shown in figures as column bars, and two conclusions can be drawn. First, predictions of $E_{z z}$ are more accurate than that of $E_{x x}$, which accounts for the straightness of the Z tows. Second, the curved beam model reveals fine stability in relative deviation compared to the FEM model. Within a large variation of the constitutive material properties, the relative deviation maintained less than $4 \%$, which indicates that the curved beam model is not sensitive to variation of the constitutive material properties and can be applicable to composites with other materials. In contrast, other analytical models are sensitive to constitutive properties on a different level. This could lead to a conclusion that the curved beam model is consistent with actual conditions of the material.

Because of the complexity of the weaving structure, the relationship between the moduli of composites and several constitutions is usually nonlinear. The results of the FEM model illustrated this argument. However, the iso-stress, iso-strain, and bridging models are essentially linear models based on the classical laminated theory; their predictions are linear with the constitutive properties. Yet the curved beam model discards linear assumptions and uses variation methods in a mechanic process. As shown in Figures 16 and 17, the curved beam model gives fine predictions of nonlinearity similar to the FEM model. It is argued that the curved beam model is a nonlinear model and could manifest fine predictions accurately and stably.

\subsection{Effect of weave parameters on elastic properties}

\subsubsection{Type of weave}

The various types of weaves can be identified by geometrical quantities $n_{g}$, which indicate a wrap/fill thread is interlaced with every $n_{g}$-th fill/wrap thread. As defined by their $n_{g}$ values, the fabrics of Figures 18 and 2 are known as plain-weave $\left(n_{g}=2\right)$, twill weave $\left(n_{g}=3\right)$, five-harness satin $\left(n_{g}=5\right)$, and eight-harness satin $\left(n_{g}=8\right)$. In this paper, the analytical and FEM models are built for these weave types via approaches demonstrated in Sections 2 and 3. 

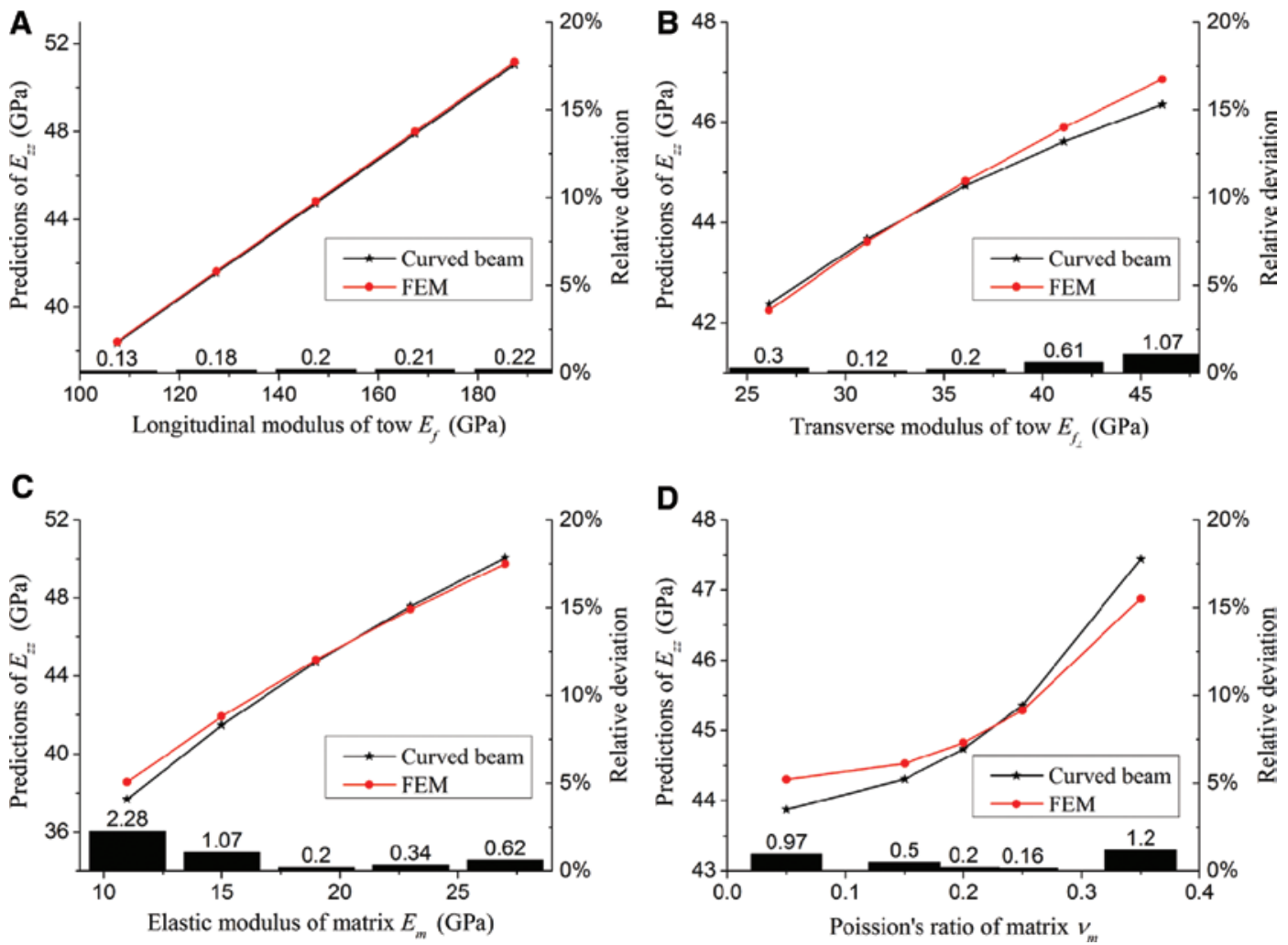

Figure 17: The predicted $E_{z z}$ (lines with left axis) and relative deviation of curved beam model (column bars with right axis) from different models with variant constitutive properties.

(A) Longitudinal modulus of tow $E_{f}$ (B) Transverse modulus of tow $E_{f_{\perp}}$. (C) Matrix elastic modulus $E_{m}$. (D) Poisson's ratio of matrix $v_{m}$.
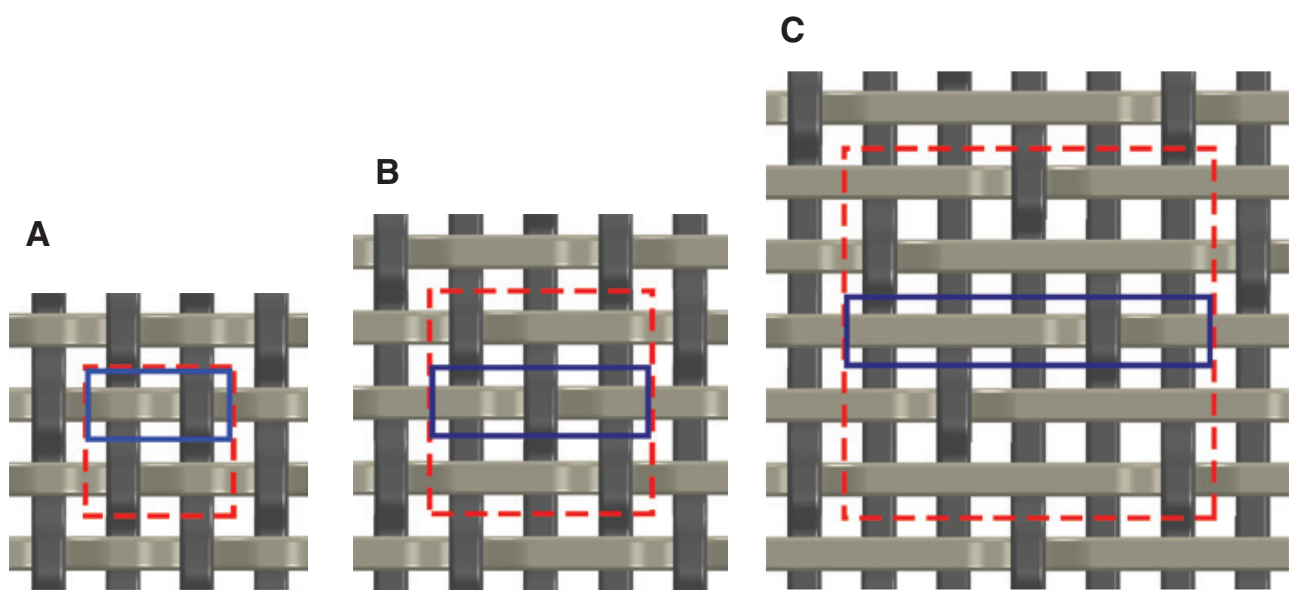

Figure 18: Woven fabrics with their RUC (in dashed line box) and ORUC (in solid line box).

(A) Plain weave $\left(n_{g}=2\right)$. (B) Twill weave $\left(n_{g}=3\right)$. (C) Five-harness satin $\left(n_{g}=5\right)$.

The predictions of elastic properties from the curved beam (analytical) and FEM models are given in Figure 19. It can be concluded that there is a positive correlation between $n_{g}$ and the elastic moduli, and the moduli grow slower with the increase in $n_{g}$ because as $n_{g}$ increases, there would be less undulated yarns, which is the main cause of stiffness loss. The stiffness, by inference, should get close to an upper limit when $n_{g}$ is large enough. The Poisson's ratio shows an inverse correlation with $n_{g}$ and approaches a lower limit when $n_{g}$ decreases. The curved beam model gives fine predictions for elastic moduli both in-plane and out-of-plane directions, while the predictions of Poisson's ratio show an ascending deviation when $n_{g}$ decreases. 

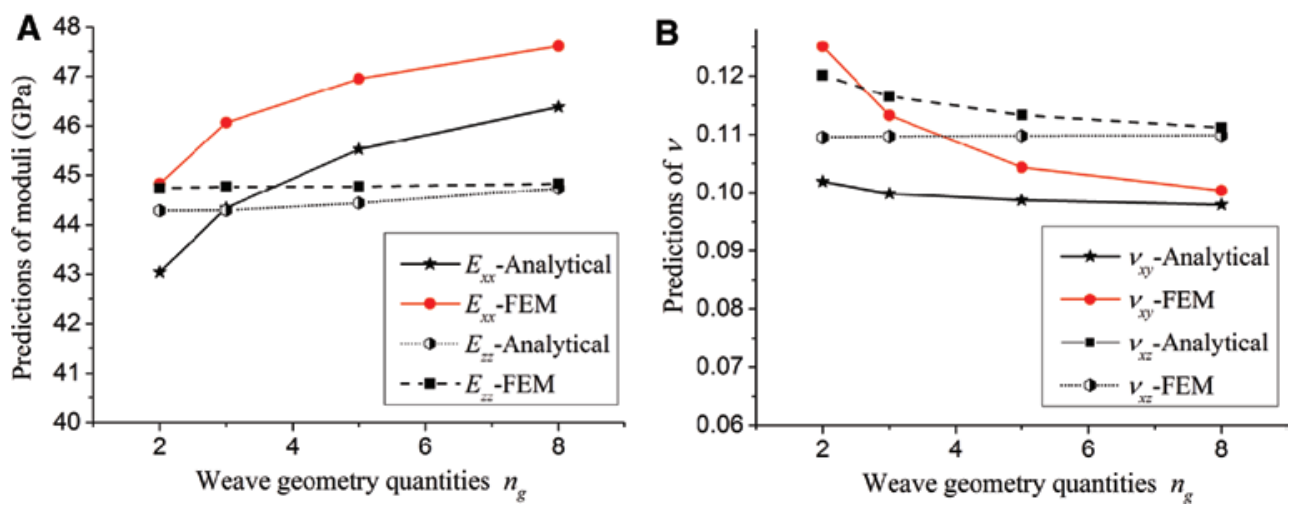

Figure 19: Effect of weave type on predicted values.

(A) Effect of weave type on elastic moduli. (B) Effect of weave type on Poisson's ratio.
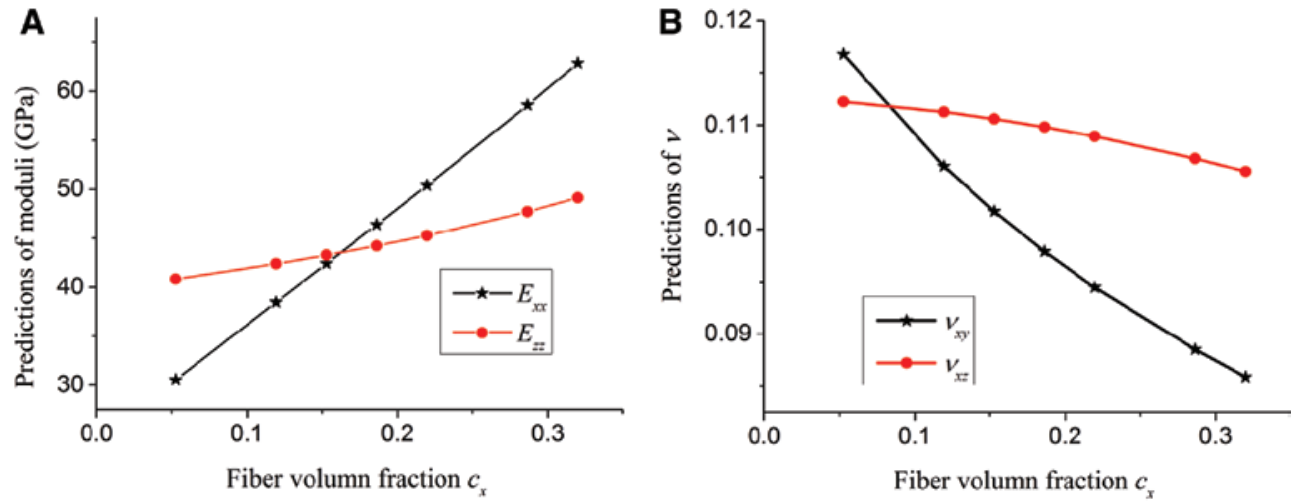

Figure 20: Effect of $c_{x}$ on predicted values.

(A) Effect of $c_{x}$ on elastic moduli. (B) Effect of $c_{x}$ on Poisson's ratio.
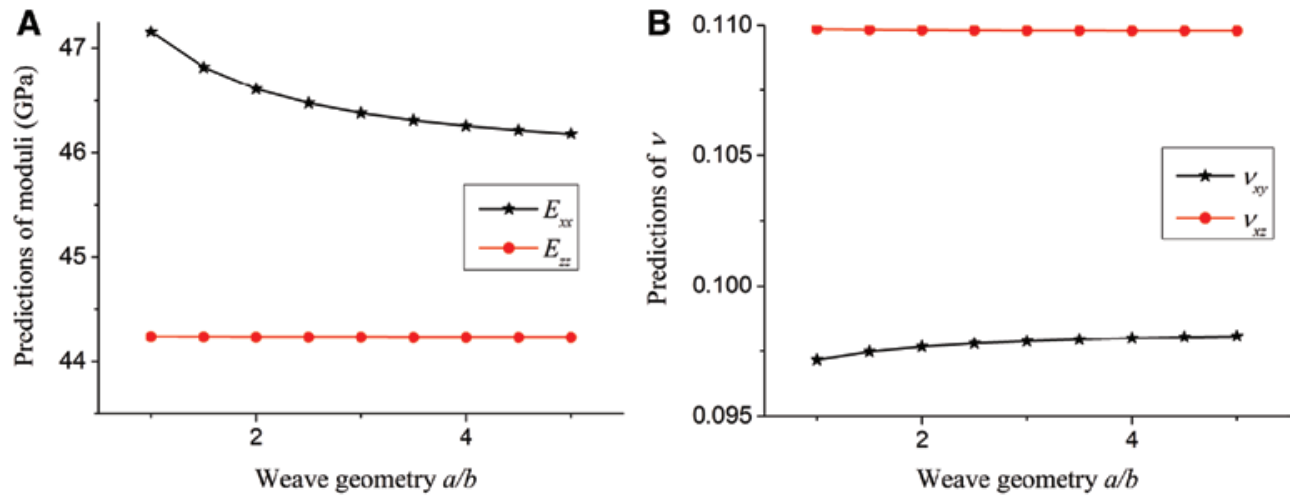

Figure 21: Effect of $a / b$ on predicted values.

(A) Effect of $a / b$ on elastic moduli. (B) Effect of $a / b$ on Poisson's ratio.

\subsubsection{Geometric parameters}

In order to appraise the effect of the geometry parameters of the braiding yarns on the elastic properties of composites, the analysis was conducted to discuss the variation of elastic constants with several parameters, which are fiber volume fraction of the $\mathrm{X}$ tow $\left(c_{x}\right)$, aspect ratio $(a / b)$, undulation length $(g)$, and depth $(h)$.

Figure 20 shows the variation of predicted elastic properties with the fiber volume fraction. It should be noted that the fabric is still balanced woven and $c_{z}$ is invariant. Compared to $E_{x x}$ and $v_{x y}$, it is obvious that $c_{x}$ has less effect on $E_{z z}$ 

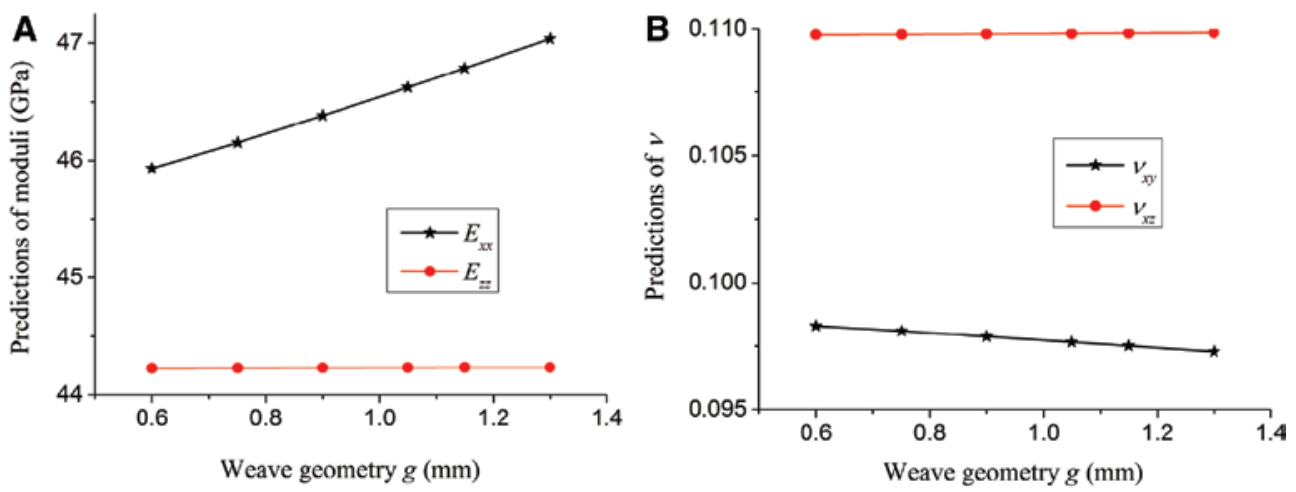

Figure 22: Effect of $g$ on predicted values.

(A) Effect of $g$ on elastic moduli. (B) Effect of $g$ on Poisson's ratio.
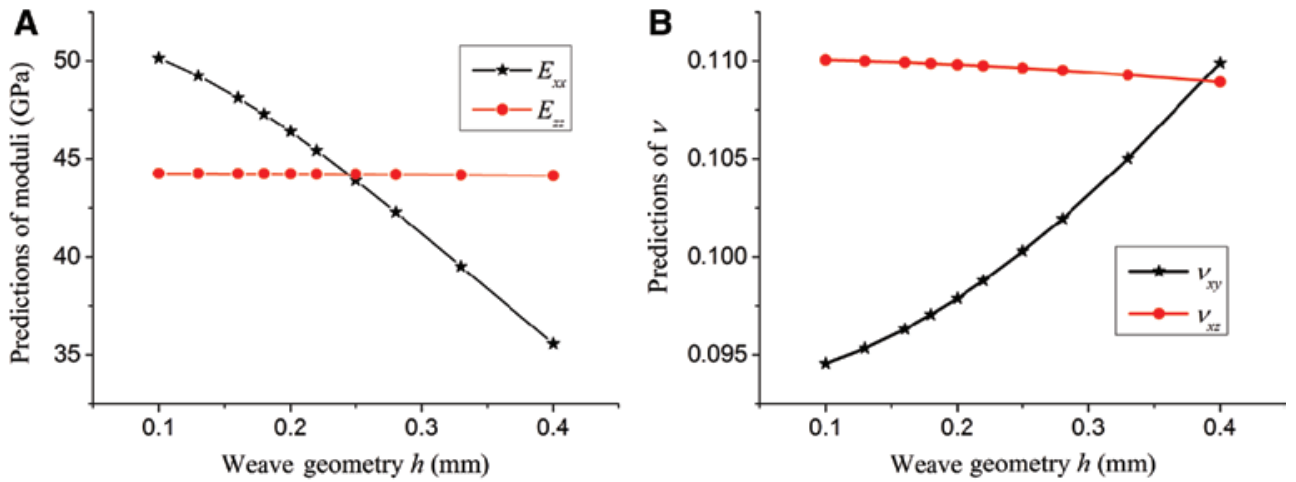

Figure 23: Effect of $h$ on predicted values.

(A) Effect of $h$ on elastic moduli. (B) Effect of $h$ on Poisson's ratio.

and $v_{x z}$. The $E_{x x}$ grow linearly as $c_{x}$ increases, and $E_{z z}$ grows slower than $E_{x x}$, but the growth rate shows a slight increment as $c_{x}$ increases. Therefore, the fiber volume fraction has a great effect on in-plane properties and limited effect on out-of-plane properties.

Figure 21 shows the variation of the predicted elastic properties with the aspect ratio $a / b$. The model was set to maintain the volume fraction ratio of each component. It is not difficult to find that $a / b$ almost has no effect on $v_{x y}$ and $v_{x z}$. It is clear that $a / b$ has little effect on $E_{z z}$ and weak effect on $E_{x x}$. With the decreasing $a / b, E_{x x}$ begins to increase gradually. Thus, it can be concluded that the effect of the aspect ratio on elastic properties can be neglected when $a / b$ is greater than 3 .

The effects of the undulation length ( $g$ ) and depth (h) on the elastic properties are given in Figures 22 and 23. It can be analyzed that the undulation parameters have an obvious effect on in-plane properties, and almost no effect on out-of plane properties. Concerning the in-plane properties, $E_{x x}$ and $v_{x y}$ show an opposite trend responding to $g$ and $h$. Increasing $g$ should diminish the inclination of woven tows, leading to the stiffness increase. Increasing $h$ leads to a wider gap between the central lines of the woven tows, and larger inclination. In order to prove the stiffness of fabrics, a wider undulation length and minor depth are needed, requiring a topological optimization for weave geometry.

\subsubsection{Influence of $Z$ tows on in-plane tensile modulus}

Micro-CT graphs in this paper show that the $\mathrm{Z}$ tows are nearly straight in composites. The $\mathrm{Z}$ tows mainly exist to enhance the strength and stiffness between fabric layers. The influence of the $\mathrm{Z}$ tows on in-plane tensile modulus

Table 7: The influence of $Z$ tows.

\begin{tabular}{lrrr}
\hline Models & Z tow & Z tow absent & Distinction \\
\hline FEM model & 47.606 & 44.684 & $6.14 \%$ \\
Modified beam & 46.383 & 43.180 & $6.91 \%$ \\
Relative error & $2.57 \%$ & $3.37 \%$ & - \\
\hline
\end{tabular}


A

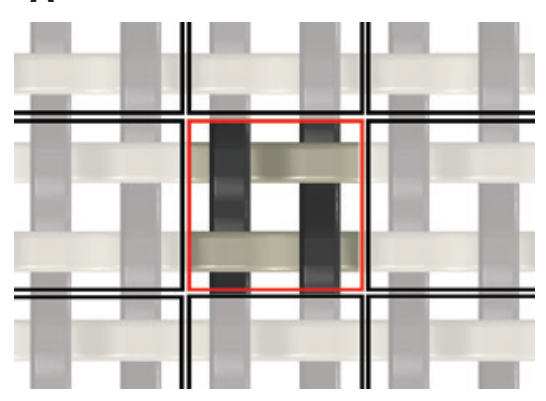

B

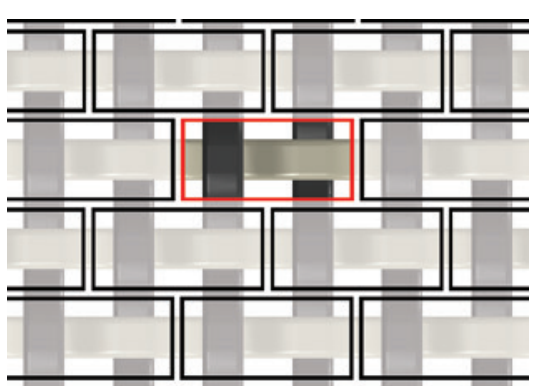

Figure 24: RUC and ORUC selected for plain weave composites.

(A) RUC. (B) ORUC.

is studied in this section. Usually, when composites are 2D fabrics, the density of the in-plane tows are higher than that in the 3D composites. In this paper, this effect is neglected to verify the influence of the $Z$ tow existence. Compared to 3D composites, only $Z$ tows will be removed and replaced with a matrix, and the other components are unchanged. Both FEM and curved beam model are employed to predict the moduli $E_{x x}$, and the results are shown in Table 7.

As shown in Table 7, the absence of the $Z$ tows brings down the in-plane elastic tensile modulus; both the FEM and curved beam model showed a decrease of $6-7 \%$. With the absence of the $\mathrm{Z}$ tows, predictions of the curved beam model is still in fine agreement with the FEM model, which indicates that the modified model is available in predicting 2D composites.

\section{Conclusion}

An offset representative unit cell (ORUC) is introduced to predict the elastic properties of the 3D eight-harness satin weave composites both analytically and numerically. The analytical model takes the interlacing force between tows and effects of the matrix supporting and Poisson's ratio into consideration, which derived the elastic properties of the unit cell directly based on the minimum complementary energy principle. In the numerical model, periodic boundary conditions for the ORUC model is derived based on previous work, and the engineering constants of the composites are obtained through a master node loading method. The experiment of the simulated material is performed under tensile test. Close correlation is obtained between experimental data and predictions.

The sensitivity study shows that within a large variation of constitutive material properties, the curved beam model derives close predictions comparing to finite element model, which indicates the validity of this model. It is also argued that the curved beam model is a nonlinear model, which could manifest fine predictions accurately and stably, and is recommended for the prediction of the elastic properties of the satin weave composite. The development of these mechanical models will provide increased efficiency in selecting the best fabric type and reinforcement for structural composites.

\section{Appendix A}

\section{Form verification study}

The form verification study is conducted to verify the validity of periodic boundary conditions for the offset representative unit cell (ORUC). Two FEM models were built by the ORUC and RUC methods, respectively, for identical plain weave composites as shown in Figure 24. The first model is the traditional RUC, which has two longitudinal

Table 8: The influence of $Z$ tow.

\begin{tabular}{lrrrr}
\hline Loads & $\boldsymbol{\sigma}_{\max } / \mathrm{MPa}$ & $\boldsymbol{\sigma}_{\min } / \mathrm{MPa}$ & $\boldsymbol{\varepsilon}_{\max } / 10^{-6}$ & $\boldsymbol{\varepsilon}_{\min } / 10^{-6}$ \\
\hline$\overline{\sigma_{x x}}$ & $189.61 / 189.47$ & $13.94 / 13.94$ & $2020 / 2010$ & $565 / 597$ \\
$\overline{\sigma_{y y}}$ & $174.28 / 174.28$ & $13.95 / 13.95$ & $1770 / 1770$ & $634 / 634$ \\
$\overline{\sigma_{z z}}$ & $147.57 / 147.57$ & $17.76 / 17.76$ & $1480 / 1480$ & $715 / 715$ \\
$\overline{\sigma_{x y}}$ & $29.27 / 29.27$ & $11.75 / 11.75$ & $887 / 887$ & $273 / 317$ \\
$\overline{\sigma_{y z}}$ & $29.74 / 29.74$ & $10.81 / 10.81$ & $936 / 936$ & $266 / 266$ \\
$\overline{\sigma_{z x}}$ & $31.62 / 31.57$ & $9.88 / 10.30$ & $942 / 942$ & $265 / 268$ \\
\hline
\end{tabular}


A

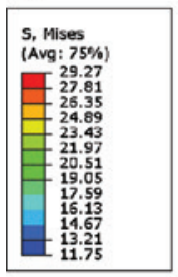

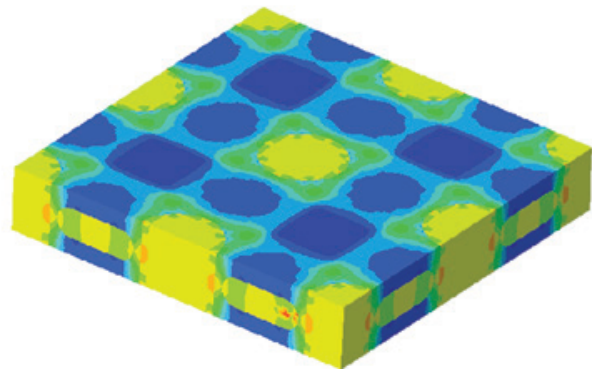

B

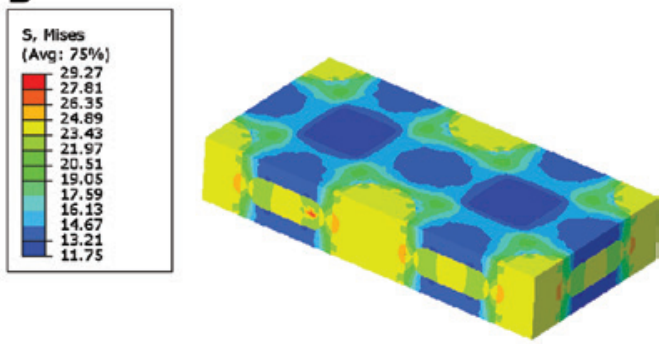

Figure 25: Stress nephogram of two models under $\overline{\sigma_{x y}}$ load.

(A) Stress nephogram of RUC model. (B) Stress nephogram of ORUC model.

and two transverse tows; the second model, ORUC, is half the size of RUC.

Periodic boundary conditions and external loads were applied to models, respectively, according to the methods demonstrated in Sections 3.2 and 3.3. If two models are equal in mechanical analysis, they should show an identical response for equivalent loads. Verification was performed by comparing strain and stress fields in two models under several load conditions.

Table 8 lists the maximum and minimum stress/strain of two models under different loads. It could be concluded that for each parameter that two models showed pretty close prediction. These differences could be a result of numerical computation precision. Figure 25 is the stress nephograms of two models under the $\overline{\sigma_{x y}}$ load. It is obvious that the two nephograms showed similar stress distribution. It is argued that the two models should be equivalent in mechanical analysis.

\section{References}

[1] Yin J, Xiong X, Zhang H, Huang B. Carbon 2006, 44, 1690-1694.

[2] McWilliams B, Yu J, Klier E, Yen C-F. Mater. Sci. Eng.: A 2014, 590, 21-29.

[3] Lu Y, Wang Y, Shen H, Pan Z, Huang Z, Wu L. Mater. Sci. Eng.: A 2014, 590, 368-373.

[4] Wei L, Meng S, Xu C, Qi F, Tian X. Adv. Compos. Mater. 2013, 22 , 165-174.

[5] Rolindk P. Carbon 1973, 11, 690.

[6] Wu Z], Brown D, Davies JM. Compos. Struct. 2002, 56, 407-412.

[7] Dong J, Huo N. Compos. Struct. 2016, 152, 1-10.

[8] Zhang D, Hayhurst DR. Int. J. Solids Struct. 2014, 51, 3762-3775.

[9] Scida D, Aboura Z, Benzeggagh ML, Bocherens E. Compos. Sci. Technol. 1999, 59, 505-517.

[10] Hahn HT, Pandey R. J. Eng. Mater. Technol. 1994, 116, 517.
[11] Aitharaju VR, Averill RC. Compos. Sci. Technol. 1999, 59, 1901-1911.

[12] Angioni S, Meo M, Foreman A. Compos. Part B: Eng. 2011, 42, 181-189.

[13] Zhou XY, Gosling PD, Pearce C), Ullah Z, Kaczmarczyk L. Int. J. Solids Struct. 2016, 80, 368-380.

[14] Song J, Wen W, Cui H, Zhang H, Xu Y. Appl. Compos. Mater. 2016, 23, 29-44.

[15] Song J, Wen W, Cui H, Zhang H, Xu Y. Appl. Compos. Mater. 2016, 23, 45-69.

[16] Prodromou AG, Lomov SV, Verpoest I. Compos. Struct. 2011, 93 , 1290-1299.

[17] Ishikawa T, Chou T. J. Mater. Sci. 1982, 17, 3211-3220.

[18] Chou TW, Ishikawa T. AIAA J. 1983, 21, 1714-1721.

[19] Hallal A, Younes R, Fardoun F, Nehme S. Compos. Struct. 2012, 94, 3009-3028.

[20] Gommers B, Verpoest I, Van Houtte P. Acta Mater. 1998, 46, 2223-2235.

[21] Tong L, Tan P, Steven GP. J. Reinf. Plast. Compos. 2002, 21, 153-173.

[22] Sheng SZ, Hoa SV. J. Compos. Mater. 2001, 35, 1701-1729.

[23] Xiong JJ, Shenoi RA, Cheng X. Compos. Part B: Eng. 2009, 40, 776-783.

[24] Cheng X, Xiong J. Compos. Struct. 2009, 88, 296-303.

[25] Hofstee J, de Boer H, van Keulen F. Compos. Sci. Technol. 2000, 60, 1041-1053.

[26] Mu J, Guan Z, Bian T, Li Z, Wang K, Liu S. Appl. Compos. Mater. 2014, 21, 773-787.

[27] Guan Z, Mu J, Su F, Bian T, Huang Y, Li L. Appl. Compos. Mater. 2015, 22, 251-267.

[28] Rao MV, Mahajan P, Mittal RK. Compos. Struct. 2008, 83, 131-142.

[29] Tang C, Sheikh MA, Hayhurst DR. J. Multiscale Model. 2010, 02, 107-126.

[30] Blacklock M, Hayhurst DR. J. Appl. Mech. 2012, 79, 051020.

[31] Reuss A. Z. Angew. Math. Mech. 1929, 9, 49-58.

[32] Suquet PM. In Homogenization Techniques for Composite Media, Springer-Verlag: France, 1987, pp. 193-278.

[33] Xia Z, Zhang Y, Ellyin F. Int. J. Solids Struct. 2003, 40, 1907-1921.

[34] Tang C, Blacklock M, Hayhurst DR. J. Compos. Mater. 2011, 45, 1461-1482.

[35] Sheikh MA, Taylor SC, Hayhurst DR, Taylor R. Model. Simul. Mat. Sci. Eng. 2001, 9, 7-23. 